\section{ORIGINAL RESEARCH}

\author{
K.D. Krueger
}

V.M. Haughton

S. Hetzel

\title{
Peak CSF Velocities in Patients with Symptomatic and Asymptomatic Chiari I Malformation
}

BACKGROUND AND PURPOSE: PCMR is used to evaluate the Chiari I malformation. We compared quantitative PCMR in symptomatic and asymptomatic patients with Chiari I.

\begin{abstract}
MATERIALS AND METHODS: PCMR image data in an axial section near the foramen magnum in a consecutive series of patients with Chiari I malformations were evaluated. Patients were classified as symptomatic for a Chiari I if they had apnea spells and/or exertional headaches and as asymptomatic if they had symptoms not considered specific for a Chiari I malformation. The PCMR CSF flow study was obtained with the same protocol for all patients and with the neck in neutral, flexed, and extended positions. Images were inspected for CSF flow jets and synchronous bidirectional flow. Peak CSF flow velocities were calculated with commercial software. Differences between the 2 groups were tested with mixed-effects ANOVA and Wilcoxon rank sum or Fisher exact probability tests with significance set at the .05 level.
\end{abstract}

RESULTS: Twenty-six patients with Chiari I were classified as symptomatic, and 24, as asymptomatic. Abnormal flow jets tended to occur more often in the symptomatic than in the asymptomatic patients $(P=.054)$. Peak CSF velocities ranged from 2 to $20 \mathrm{~cm} / \mathrm{s}$ in the symptomatic and the asymptomatic groups and did not differ significantly between the 2 groups or with neck position.

CONCLUSIONS: Peak CSF flow velocities near the foramen magnum did not differentiate symptomatic and asymptomatic patients with Chiari I.

ABBREVIATIONS: ANOVA = analysis of variance; CFD = computational flow dynamics; $\mathrm{nA}=$ number asymptomatic; $\mathrm{nS}=$ number symptomatic; PCMR = phase-contrast MR imaging

$\mathbf{F}_{1}$ iede and Roesmann, ${ }^{1}$ who first described the "adult Chiari malformation," noted that the malformation may be symptomatic in some individuals and asymptomatic in others. Because of the large variety of symptoms that may characterize the malformation and because of the relative lack of specific signs and symptoms, clinical classification of patients with Chiari I as symptomatic or asymptomatic is based on clinical judgments. An objective test would facilitate the selection of patients who would benefit from craniovertebral decompression. The position or the shape of the tonsils in the spinal canal and the degree of tonsil pegging do not predict the presence or severity of clinical symptoms. ${ }^{2}$ Therefore, PCMR has a role in the evaluation of patients with Chiari I, especially those who are candidates for craniovertebral decompression to relieve symptoms and syringomyelia.

Many PCMR studies have documented that the cyclic

\section{Received March 16, 2010; accepted after revision April 15.}

From the University of Wisconsin (K.D.K.), Madison, Wisconsin; University of Wisconsin School of Medicine and Public Health (V.M.H.), Madison, Wisconsin; and Department of Biostatistics and Medical Informatics (S.H.), University of Wisconsin, Madison, Wisconsin.

This work was supported in part by the University of Wisconsin Institute for Clinical and Translational Research funded through a National Institutes of Health Clinical and Translational Science Award, grant 1 UL1 RR025011. Victor Haughton was supported by a grant from the Center of Excellence of the Norwegian Research Council to the Center for Biomedical Computing at Simula Research Laboratory, Lysaker, Norway.

Please address correspondence to Victor Haughton, MD, University of Wisconsin School of Medicine, Department of Radiology, E3/311 Clinical Science Center, 600 Highland Ave, Madison, WI 53792; e-mail: vmhaughton@wisc.edu

Indicates open access to non-subscribers at www.ajnr.org

DOI 10.3174/ajnr.A2268 cardiac-related CSF flow in the foramen magnum differs in velocity and wave form between patients with Chiari I and control subjects. ${ }^{3-11}$ Fewer studies have been published in which CSF flow has been compared in symptomatic and asymptomatic subjects with Chiari I. In 1 study, the accuracy of PCMR images, interpreted by neuroradiologists qualitatively to distinguish the "symptomatic" from the "asymptomatic" or incidental tonsillar malformations, was approximately $75 \%$ with both false-positive and false-negative studies. ${ }^{12}$ The goal of this study was to determine if quantitation of CSF flow at the foramen magnum improves the differentiation of the symptomatic and asymptomatic patients with Chiari I. We chose to compare peak systolic and diastolic CSF velocities, which differ significantly between patients with Chiari I and controls and which reflect the degree of flow inhomogeneity. We elected to include measurements of peak velocities with the subject's neck in neutral, flexed, and extended positions, to increase the statistical power of the study and to test if CSF flow velocities vary with the neck flexion and extension.

\section{Materials and Methods}

The institutional review board of the University of Wisconsin Hospital and Clinics approved our Health Insurance Portability and Accountability Act-compliant retrospective study and granted a waiver of informed consent.

\section{Patient Selection}

A spreadsheet is maintained by the Pediatric Specialty Clinic containing data on patients with the diagnosis of a Chiari I malformation, either discovered incidentally during a CT or MR imaging examination or documented in a symptomatic patient evaluated for treatment 


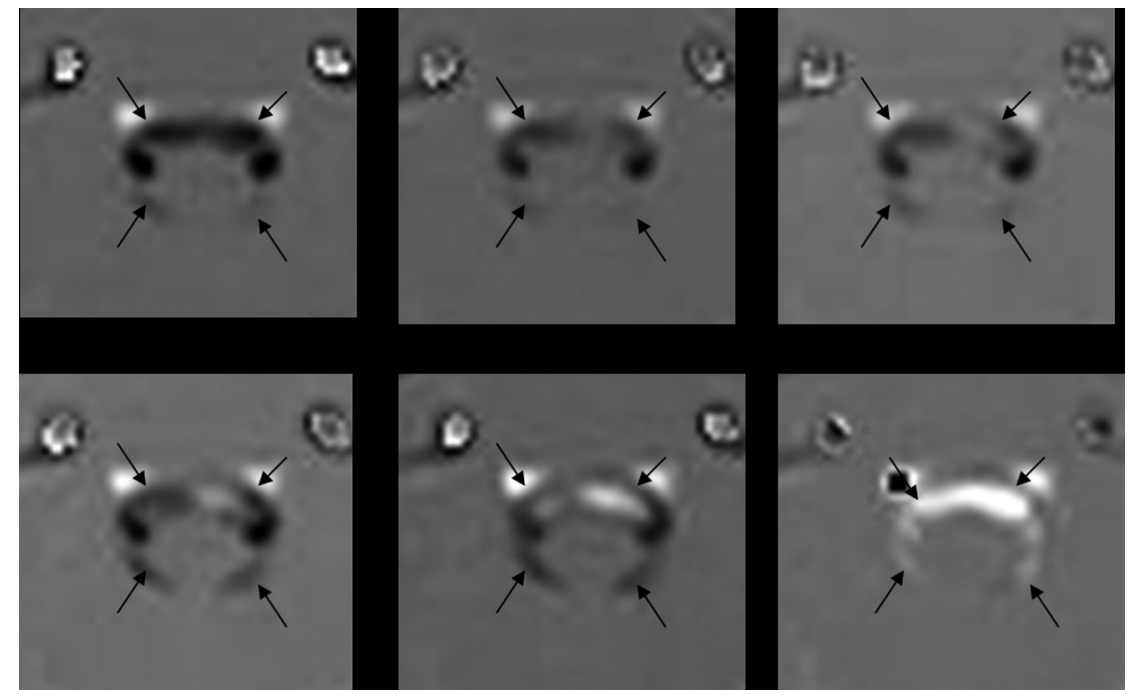

Fig 1. From top left to lower right, PCMR images of 6 of 14 successive phases in a cardiac cycle in a patient with symptomatic Chiari I malformation and evidence of synchronous bidirectional flow. The CSF subarachnoid space is indicated with black arrows. Flow changes during these 6 phases go from a negative (black) to a positive direction (white). During the final 3 phases in the series, flow is evident in both positive and negative directions.

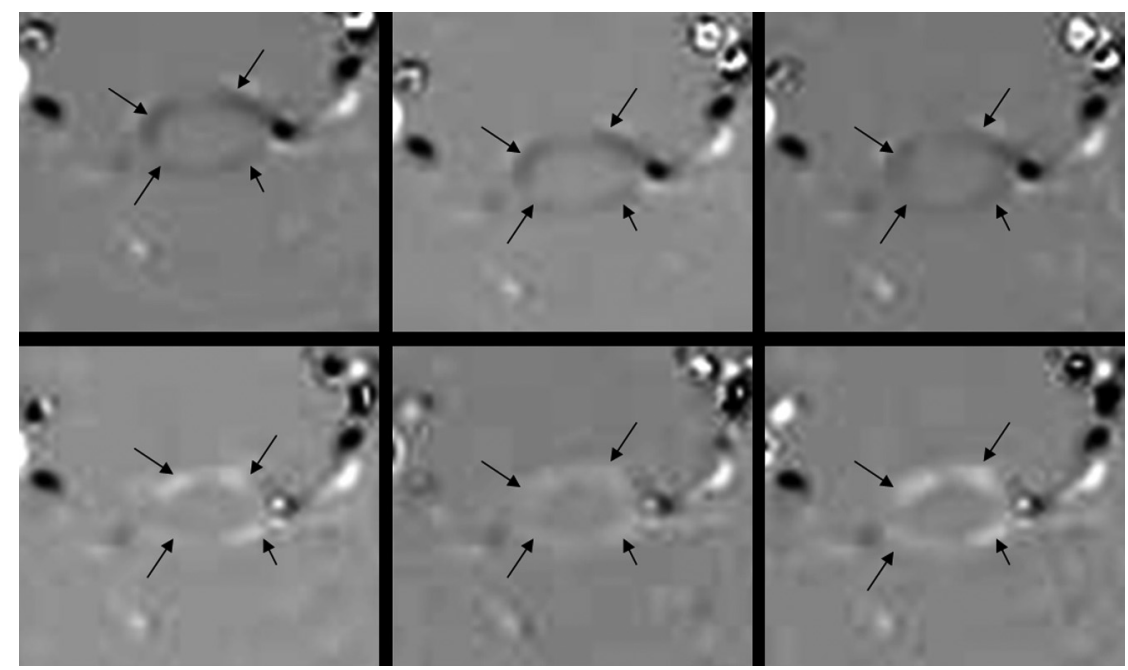

Fig 2. From top left to lower right, PCMR images of 6 of 14 successive phases in a cardiac cycle in a patient with asymptomatic Chiari I malformation and no evidence of jets. The arrows demonstrate the subarachnoid space. The CSF flow changes during these 6 phases go from a negative direction (upper 3 images) to a positive direction (lower 3 images). At no time in the cardiac cycle are large jets evident.

options. Each patient seen in this clinic had a thorough history, physical examination, and neurologic examination, on the basis of which the diagnosis and classification were made and treatment was recommended. In the spreadsheet, patient age, syrinx location and length, and tonsillar extension into the cervical spinal canal were tabulated. The presence of apnea spells, Valsalva or exertional headaches, other headaches, back or neck pain, numbness or weakness in extremities, difficulty swallowing, cranial nerve dysfunction, ataxia, scoliosis and subsequent craniovertebral decompression were tabulated if present.

To obtain a cohort of "symptomatic" and "asymptomatic" patients, we reviewed the data collected between November 30, 1999, and August 31, 2008. For the "symptomatic" classification, patients who had apnea spells or exertional headaches, considered relatively specific for the Chiari I malformation, were selected. Additional inclusion criteria were selection for craniovertebral decompression and cerebellar tonsils $\geq 5 \mathrm{~mm}$ below the foramen magnum on MR imaging. Patients with hydrocephalus or intracranial mass were excluded. For the "asymptomatic" classification, patients who lacked apnea spells or exertional headaches were selected. Additional inclusion criteria for the asymptomatic group were cerebellar tonsils $\geq 5 \mathrm{~mm}$ below the foramen magnum and not being selected for craniovertebral decompression. Exclusion criteria for this group were $\geq 2$ of the other signs or symptoms listed in the spreadsheet-that is, headaches, back or neck pain, numbness or weakness in the extremities, swallowing difficulty, cranial nerve dysfunction, ataxia, or scoliosis. Patients who did not meet inclusion and exclusion criteria for either of the 2 classifications were not included in the study.

\section{MR Imaging}

Imaging was performed on 1 of three $1.5 \mathrm{~T}$ scanners equipped with similar software and PCMR capabilities and analysis packages. Imaging included localizer images, sagittal T1- and T2-weighted images of the cervical spine, and a series of head and axial spine images as clinically indicated. Axial and sagittal PCMR images were acquired in each patient with similar techniques. The images were gated to the cardiac cycle by electrocardiography. The plane of acquisition for the 


\section{Abnormal Flow Jets}

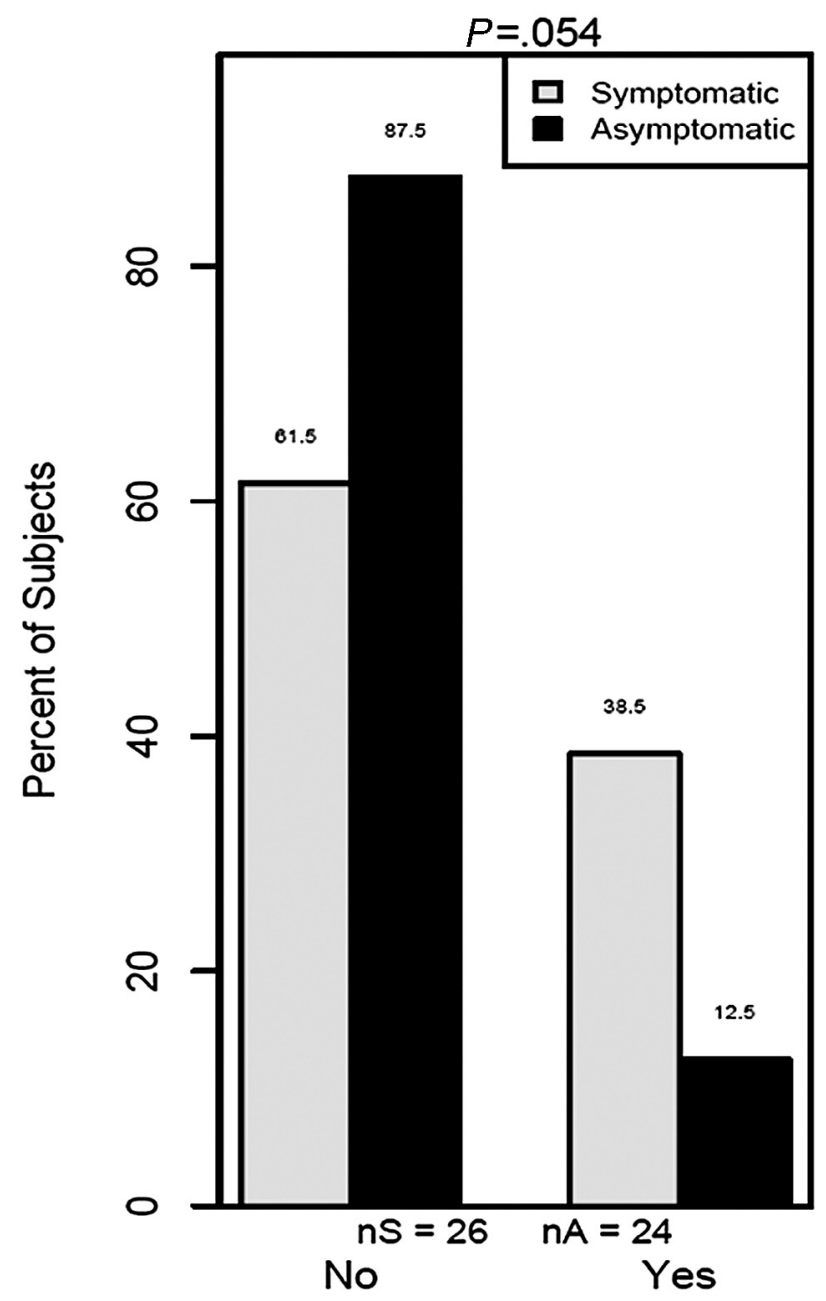

Fig 3. Percentage of symptomatic and asymptomatic patients with Chiari I with abnormal flow jets.

PCMR images was selected according to a standard protocol. For sagittal images, the midline sagittal plane was chosen. For the axial images, a plane transverse to the axis of the spinal canal immediately below the tonsillar tips was selected. For the phase-contrast flow measurement with the axial PCMR, the acquisition parameters were flip angle, $20^{\circ}$; TR/TE, 20/5 ms; section thickness, $5 \mathrm{~mm}$; FOV, $180 \mathrm{~mm}$; matrix, $256 \times 256$; and encoding velocity $10 \mathrm{~cm} / \mathrm{s}$. In each subject, the CSF flow images were first acquired with the patient's neck in a neu- tral position, then in a flexed, and finally in an extended position. Flow images were stored on the PACS of the institution.

A neuroradiologist with experience in CSF flow studies, blinded to the classification of the patient as symptomatic or asymptomatic, reviewed the PCMR studies in all patients included in the symptomatic and asymptomatic groups for evidence of CSF flow jets and synchronous bidirectional flow, signs used in previous studies to differentiate symptomatic and asymptomatic CSF flow patterns. The neuroradiologist also evaluated the position of the neck in the neutral, flexion, and extension studies to confirm that neck flexion and extension were achieved for these studies. The neuroradiologist inspected images for craniovertebral abnormalities and for the shape of the tonsils.

\section{CSF Velocity Calculation}

CSF flow velocities were calculated from the axial PCMR images on a commercial program (ReportCARD; GE Healthcare, Milwaukee, Wisconsin). The 14 successive flow images were inspected to determine their technical adequacy and the anatomic location of the vertebral arteries and subarachnoid space. A region of interest was selected on the PCMR images to include the entire subarachnoid space and exclude the vertebral arteries. A second region was selected to include only stationary tissue outside the foramen magnum, to calculate a correction of the CSF flow data for eddy currents. The ReportCARD program displayed the velocities in tabular and graphic formats for each voxel in each of the 14 phases of the cardiac cycle. All graphs were reviewed by 1 investigator to check for aliasing, jets, and possible contamination with vascular flow. The greatest positive value during the systolic phase and the greatest negative velocity during the diastolic phase were recorded as the peak systolic and diastolic velocities. Peak flow velocities were calculated similarly for flexion and extension.

\section{Statistical Testing}

A mixed-effects ANOVA model with peak flow velocities as the response; group, neck position, and their interaction as fixed effects; and subjects as a random effect to account for multiple measures was fit to examine differences in velocities due to symptomatic/asymptomatic groups and by the 3 neck positions. Model diagnostic checks verified that the assumptions of the model were sufficiently met, separately for systolic velocities and diastolic velocities.

Differences in the incidence of jets and bidirectional flow between the symptomatic and asymptomatic groups were tested for significance by a Fisher exact test with significance set at .05. Differences in flow velocities between symptomatic and asymptomatic individuals at each individual neck position were tested by 2 -sided Wilcoxon rank

\begin{tabular}{|c|c|c|c|c|c|c|c|}
\hline \multicolumn{8}{|c|}{ Average peak velocities for symptomatic and asymptomatic patients by neck position and flow direction ${ }^{a}$} \\
\hline \multirow[b]{2}{*}{$\begin{array}{l}\text { Position/Flow } \\
\text { Direction }\end{array}$} & \multicolumn{3}{|c|}{ Symptomatic } & \multicolumn{3}{|c|}{ Asymptomatic } & \multirow[b]{2}{*}{$\begin{array}{c}P \\
\text { Value }\end{array}$} \\
\hline & No. & $\begin{array}{l}\text { Mean } \\
( \pm S D)\end{array}$ & Range & No. & $\begin{array}{l}\text { Mean } \\
( \pm S D)\end{array}$ & Range & \\
\hline \multicolumn{8}{|l|}{ Neutral } \\
\hline Systolic & 23 & $6.0(2.53)$ & $(2.4-11.7)$ & 22 & $6.6(3.07)$ & $(1.9-13.4)$ & .570 \\
\hline Diastolic & 23 & $4.4(2.24)$ & $(1.5-10.9)$ & 22 & $4.4(1.61)$ & $(0.9-7.7)$ & .525 \\
\hline \multicolumn{8}{|l|}{ Flexion } \\
\hline Systolic & 21 & $5.9(3.27)$ & $(2.1-12.0)$ & 16 & $5.7(3.17)$ & $(2.4-15.3)$ & .963 \\
\hline Diastolic & 21 & $4.2(2.35)$ & $(1.9-9.2)$ & 16 & $3.9(2.19)$ & $(0.7-10.3)$ & .842 \\
\hline \multicolumn{8}{|l|}{ Extension } \\
\hline Systolic & 23 & $7.1(3.93)$ & $(2.0-8.9)$ & 17 & $5.5(2.33)$ & $(2.3-10.1)$ & .256 \\
\hline Diastolic & 23 & $4.9(2.52)$ & $(1.6-9.5)$ & 17 & $4.1(1.24)$ & $(2.0-5.9)$ & .511 \\
\hline
\end{tabular}

annits for the means and range values are centimeters per second. 
sum tests. Before these tests were run, the distributions were examined and were deemed borderline normally distributed; therefore, a nonparametric test was used. All analysis was conducted by using R-project 2.8.1 (http://r-project.en.softonic.com).

\section{Results}

\section{Patients}

In the time interval selected for the study, 156 patients were tabulated. Of these, 29 were excluded for 1 of the following reasons: tonsils did not extend $\geq 5 \mathrm{~mm}$ below the foramen magnum, no flow study was performed (8 patients), or flow data were not suitably archived for CSF flow calculations (17 patients). Of the remaining 127 patients, 26 were classified as symptomatic and 24 as asymptomatic. The 26 patients classified as symptomatic according to our criteria included 8 males and 18 females, $1-54$ years of age (mean age, 17 years). The 24 patients classified as asymptomatic included 6 males and 18 females, 3-43 years of age (mean age, 14 years). Seven patients in the asymptomatic group had no symptoms, and 17 had nonspecific headache symptoms. Seventy-seven patients did not meet the inclusion and exclusion criteria and were not included in the study. They included 49 patients who had symptoms tabulated in the spreadsheet but were not selected for surgical treatment and, therefore, did not meet criteria for "symptomatic." They also included 28 patients who were not selected for surgical treatment and had $\geq 1$ symptom associated with the Chiari I malformation and, therefore, did not qualify as "asymptomatic."

\section{MR Imaging}

All patients included in the study had PCMR images adequate to evaluate CSF flow patterns. Flow jets or synchronous bidirectional flow was present in 12 of 26 symptomatic patients (Fig 1) and in 6 of 24 asymptomatic patients (Fig 2). Flow jets tended to occur more often in the symptomatic than in the asymptomatic patients $(P=.054)$ (Fig 3$)$. The neuroradiologist noted no differences in tonsil shape or position between the symptomatic and asymptomatic groups and noted no basilar invagination or other craniovertebral anomalies in either patient group.

\section{CSF Velocities}

Some patients lacked complete velocity data for the 3 neck positions. Twenty-three symptomatic and 22 asymptomatic patients had systolic and diastolic data for the neutral position, 21 symptomatic and 16 asymptomatic patients had systolic and diastolic data for the flexion position, and 23 symptomatic and 16 asymptomatic patients had systolic and diastolic data for the extension position. Missing data were due to incomplete data acquisition or because of technical error. The average velocities for the 3 positions are shown in the Table. Assuming that data were missed randomly, we found no significant differences between symptomatic and asymptomatic groups or between neck positions $(P$ values $=.3-.9$, ANOVA).

\section{Discussion}

The study showed no significant differences in peak CSF velocities in the foramen magnum of patients classified on the basis of strict criteria as having "symptomatic" Chiari I malformation in comparison with those classified as "asymptom- atic." Neck position did not have a significant effect on peak CSF velocities. The study suggests that to differentiate symptomatic and asymptomatic patients with Chiari I, quantification of CSF flow at the foramen magnum adds little to the visual inspection of flow patterns.

Qualitative and quantitative results in this study agree with those in previous publications. The presence of greater jets and synchronous bidirectional flow in symptomatic-versusasymptomatic patients with Chiari I in this study concurs with findings previously reported. ${ }^{11,12} \mathrm{We}$ found CSF velocities in the range of $2-20 \mathrm{~cm} / \mathrm{s}$ in patients with Chiari I, as in previous studies. ${ }^{4,6,11}$ To our knowledge, no previous study has quantitatively compared the peak CSF velocities and flow patterns of symptomatic and asymptomatic patients with Chiari I.

The most significant limitation in this study is the lack of a criterion standard to classify and distinguish symptomatic and asymptomatic patients with Chiari I. We relied on rigid criteria, which excluded the patients on whom we had CSF flow data. Our groups cannot be considered typical or representative of symptomatic and asymptomatic patients with Chiari I in general because of the strictness of our criteria. Some cases may have been misclassified. Other limitations include the lack of matching of age and sex between the 2 groups. The younger ages of the symptomatic patients may have biased the results toward greater velocities in this group. ${ }^{13}$ Some subjectivity in the recognition of flow jets must be acknowledged, though the image inspection was performed in a blinded manner and as in a previous study. ${ }^{12}$ The degree of flexion and extension was not measured or compared between groups in this study. In both groups, flexion and extension were limited and variable in the environment of the scanner.

This study suggests that measuring CSF velocities at the foramen magnum does not significantly add to the differentiation of symptomatic and asymptomatic patients with Chiari I. Quantitation of flow at the foramen magnum does not predict whether a patient will benefit from craniovertebral decompression. Whether sampling CSF velocities below the foramen magnum will improve the accuracy of classification has not been shown, while CFD studies suggest that peak velocities may increase below the foramen magnum. ${ }^{14,15}$ We await further studies with CFD to better characterize CSF flow.

\section{Conclusions}

Quantitative analysis of CSF flow velocities in the foramen magnum did not differentiate symptomatic and asymptomatic patients with Chiari I.

\section{References}

1. Friede RL, Roessmann U. Chronic tonsillar herniation: an attempt at classifying chronic herniations at the foramen magnum. Acta Neuropathol 1976;34: 219-35

2. Meadows J, Kraut M, Guarnieri M, et al. Asymptomatic Chiari type I malformations identified on magnetic resonance imaging. J Neurosurg 2000;92: 920-26

3. Wolpert SM, Bhadelia RA, Bogdan AR, et al. Chiari I malformations: assessment with phase-contrast velocity MR. AJNR Am J Neuroradiol 1994;15:1299_ 308

4. Hofman E, Warmuth-Metz M, Bendzus M, et al. Phase-contrast MR imaging of the cervical CSF and spinal cord: volumetric motion analysis in patients with Chiari I malformation. AJNR Am J Neuroradiol 2000;21:151-58

5. Armonda RA, Citrin CM, Foley KT, et al. Quantitative cine mode magnetic resonance imaging of Chiari I malformations: an analysis of cerebrospinal fluid dynamics. Neurosurgery 1994;35:214-24 
6. Heiss JD, Patronas N, DeVroom HL, et al. Elucidating the pathophysiology of syringomyelia. J Neurosurg 1999;91:553-62

7. Ellenbogen RG, Armonda RA, Shaw DW, et al. Toward a rational treatment of Chiari I malformation and syringomyelia. Neurosurg Focus 2000;8:E6

8. Alperin N, Sivaramakrishnan A, Lichtor T. Magnetic resonance imaging-based measurements of cerebrospinal fluid and blood flow as indicators of intracranial compliance in patients with Chiari malformation. J Neurosurg 2005;103: $46-52$

9. Haughton VM, Korosec FR, Medow JE, et al. Peak systolic and diastolic CSF velocity in the foramen magnum in adult patients with Chiari I malformations and in normal control participants. AJNR Am J Neuroradiol 2003;24: $169-76$

10. Levy LM. MR identification of Chiari pathophysiology by using spatial and temporal CSF flow indices and implications for syringomyelia. AJNR Am J Neuroradiol 2003;24:165-66
11. Quigley MF, Iskandar B, Quigley ME, et al. Cerebrospinal fluid flow in foramen magnum: temporal and spatial patterns at MR imaging in volunteers and in patients with Chiari I malformation. Radiology 2004;232:229-36

12. Hofkes SK, Iskandar BJ, Turski PA, et al. Differentiation between symptomatic Chiari I malformation and asymptomatic tonsillar ectopia by using cerebrospinal fluid flow imaging: initial estimate of imaging accuracy. Radiology 2007; 245:532-40. Epub 2007 Sep 21

13. Iskandar BJ, Haughton V. Age-related variations in peak cerebrospinal fluid velocities in the foramen magnum. J Neurosurg 2000;103(6 suppl):508-11

14. Roldan A, Wieben O, Haughton V, et al. Characterization of CSF hydrodynamics in the presence and absence of tonsillar ectopia by means of computational flow analysis. AJNR Am J Neuroradiol 2009;30:941-46. Epub 2009 Mar 19

15. Linge S, Haughton V, Løvgren AE, et al. Effect of tonsillar herniation on cyclic cranio-vertebral CSF fluid flow studied with computational flow analysis. AJNR Am J Neuroradiol 2010;31:185-92. Epub 2009 Sep 3 